Fecha de recepción: mayo 2021 Fecha de aceptación: junio 2021 Versión final: julio 2021

\section{Aproximaciones dialógicas transversales del Diseño. Dialogar el Diseño como disciplina científica}

Martha Gutiérrez Miranda ${ }^{(1)}$

Resumen: La epistemología del diseño ha orientado sus esfuerzos en tratar a la disciplina como objeto de estudio y encuadrarla en el marco de las ciencias. La intención principal ha sido comprenderla y hacer que la comprendan como actividad científica, la aborden como disciplina y la ubiquen en el contexto académico. De esta manera, el diseño se instituye como objeto de estudio, pero también como sujeto: histórico, social, cultural, político, semiótico y lingüístico, sobre el que subyacen supuestos básicos que permitirían reconocer su validez científica.

Como disciplina, cuyo origen se sitúa en el siglo XX, su acercamiento a las ciencias quedó ampliamente manifestado en el uso de métodos de diseño, procedimientos, tecnologías y técnicas científicas, así como en la práctica profesional y formación de diseñadores. Ha evolucionado y permitido ver que también se vale de otras ciencias, para evidenciar procesos inter o multidisciplinarios que convergen en su planteamiento. Analizando el abordaje de su praxis, como disciplina de su tiempo, se ha enmarcado en una transversalidad quizá intencional o hasta cotidiana. De esta manera atraviesa otras disciplinas para determinar supuestos que trascienden más álla del ámbito profesional y económico y permiten convertirla en un fenómeno de análisis que afecta a todos los aspectos de nuestra sociedad y de la vida cotidiana.

La relación entre el diseño y la ciencia fue planteada por Herbert Simon en la década de los setentas del siglo XX, considerando al diseño como una ciencia. Su postura expone la necesidad de estudiar científicamente el mundo creado por el hombre y mirar al diseño como una ciencia de lo artificial. Este artículo ofrece una propuesta, que analiza al proceso de diseño, sus métodos, técnicas, tecnologías y productos, para poner sobre la mesa la cientificidad del diseño y sus alcances como una disciplina que trasversaliza a las demás.

Palabras clave: diseño - diálogo - discilplina - ciencia - transversal

[Resúmenes en inglés y portugués en la página 162 ]

(1) Diseñadora de la Comunicación Gráfica, Maestra en Mercadotecnia y Doctora en Diseño, con estudios posdoctorales en Innovación, cultura y tecnología. Coordina la Maestría en Diseño y Comunicación Hipermedial, es Directora de Tecnología, Innovación y Cultura Universitaria en la Universidad Autónoma de Querétaro. Miembro del Sistema Nacional de Investigadores del CONACYT. Ha impartido docencia en Licenciatura, Especialidad, Maestría y Doctorado. Sus líneas de investigación se centran en el análisis entre el diseño y la tecnología. 


\section{Introducción}

Han sido muchos los pensadores que, de manera constante, insistieron en que la realidad debe ser comprendida de forma dialéctica; es decir, a partir de un ritus entre dialogar y discutir, para descubrir la verdad mediante la exposición y confrontación de razonamientos y argumentaciones contrarios entre sí, hasta que a través de la síntesis, se regulan esas nuevas formas de comprensión. Desde Platón y Aristóteles, pasando por Bacon, Hegel, Marx, Engels y Kant, muchos han coincidido en que el mundo no es un objeto estático, sino más bien un flujo constante, dinámico y en movimiento perpetuo, de ires y venires y, más que nunca, se comprueba esta forma de verlo, asimilarlo y sobre todo, tratar de explicarlo, lo que hace patente la importancia dialogar, como un ejercicio de pensamiento necesario.

Ante la presencia, particularmente en esta era posmoderna, de planteamientos, replanteamientos y nuevas concepciones, así como nuevas propuestas epistemológicas acerca de cómo las disciplinas científicas abordan las crisis y necesidades de conocimiento, los esfuerzos más interesantes oscilan entre la multidisiciplinariedad, interdisciplinariedad y la transdisciplinariedad, como enfoques para unificar o, más bien, cohesionar a las disciplinas, sus alcances y fronteras. Estas relaciones se caracterizan y manifiestan de diversas maneras, desde lo puramente conceptual, hasta lo totalmente procedimental y en ellas convergen sujetos y objetos de estudio, en un diálogo constante.

$\mathrm{El}$ acercamiento intencional de este documento pone el acento en tratar de identificar de forma generalizada las relaciones dialógicas entre el diseño y sus métodos, técnicas y aproximaciones sistemáticas, acercándolo al ámbito de la ciencia. Para iniciar con el planteamiento medular de este texto, se precisa hacer una ineludible salvedad conceptual de los términos: epistemología, ciencia, transdisciplinariedad y transversalidad, que permitirán enmarcar las intencionalidades de ésta reflexión y a su vez relacionarlas con el Diseño.

\section{Aceramiento a los conceptos}

En sentido general, para la mayor parte de los pensadores griegos, especialmente para Platón y Aristóteles el término episteme significaba entender algo, saber o conocer, esto lo ha convertido en la raíz de muchos términos propios de la mayoría de las teorías sobre el conocimiento. De forma semejante, otro concepto que tomó un significado muy similar en la cultura greco-latina, fue gnosis, que alude también al conocimiento, éste llegó a nosotros directamente del latín cognoscere, que - a su vez- remitía al griego gignoskein, que hace refererencia al proceso de llegar a conocer o saber.

Ambos términos, eptisteme y gnosis, evidencian la esencia del concepto de "conocimiento", sin embargo, con el paso del tiempo sufrieron transformaciones y adquirieron connotaciones distintas. Es a James Frederick Ferrier (1808-1864) a quien se le atribuye la autoría sobre el término moderno de epistemología, mismo que utilizó por vez primera en su texto titulado Institutes of Metaphysic (1854). Este pensador enfatizó que la epistemología no es más que la doctrina sobre el conocimiento científico y trata de representarla mediante 
estructuras a las que llamó teoremas lógicos, a través de los cuales, se interrelacionan e integran las disciplinas científicas. En su planteamiento inicial, propuso un sistema de ciencia que, si se revisa a cabalidad, pudiera ser el antecedente directo de los cruces disciplinares que hoy enmarcan los esfuerzos desde la interdisciplinariedad, multidisciplinariedad y la transdiciplinariedad.

En el contexto actual, hablar de la epistemología implica relacionarla con la filosofía y determinar que, de forma generalizada, su objeto de estudio es el conocimiento científico y los caminos o métodos que se toman para alcanzarlo. De forma particularizada, especifica el estudio de la teoría del conocimiento y precisa incluso distintas acotaciones, por ejemplo, la epistemología del propio diseño.

Para el caso del término disciplina, definirlo, implica revisar muchas opiniones, pues su origen además de etimológico, se asocia a las condiciones históricamente peculiares de la vida monástica durante el medioevo. Disciplina, viene del latín disciplina, derivado de la palabra discipulus, discípulo, que - a su vez- proviene del latín discere, que significa aprender. Cuando se habla de disciplina en su más pura expresión, se alude literalmente al esfuerzo que alguien hace por aprender. Y en el contexto actual, también hace referencia a los saberes que, como extensión natural y clasificación de las ciencias, abordan el conocimiento y la instrucción que debe aprenderse de forma sitematizada.

Por su parte, el término ciencia proviene del latín scientia, que también significa conocimiento. Incluye todo conocimiento ordenado o sistemáticamente organizado. Y se relaciona con scire, "conocer", así como con los términos nous o noesis, los cuales también significan saber o reconocer. Algunas definiciones puntuales de ciencia que resulta oportuno considerar, son:

conocimiento sistemático que el hombre realiza sobre una realidad determinada, lo expresa en un conjunto de proposiciones coherentes y lógicas (proposiciones teóricas) a partir de las cuales se formulan alternativas de esa realidad. (Méndez, 1998, p.8).

Y por otro:

Un conjunto de conocimientos racionales, ciertos o probables, que obtenidos de una manera metódica y verificados en su contrastación con la realidad se sistematizan orgánicamente haciendo referencia a objetos de una misma naturaleza, cuyos contenidos son susceptibles de ser transmitidos (Ander-Egg, 1995, p.33).

Interrelacionando estos planteamientos, puede decirse que el fin general de todos ellos es obtener conocimiento genuino acerca del mundo. Tal vez esto podría decirse con respecto a todas las ciencias, dentro de las que se encuentran tanto las naturales, como las sociales. Y considerando este abordaje, hoy se entiende la ciencia no sólo como un sistema de conceptos, plantemientos, teorías o tesis, también, como una forma específica de actividad social dirigida a producir, distribuir y aplicar los conocimientos sobre la naturaleza y la 
sociedad. Incluso, como lo plantea Kröber (1986), la ciencia se configura como una institución social, como un sistema de organizaciones científicas, cuya estructura y desarrollo se encuentran estrechamente vinculados con la economía, la política, con los fenómenos culturales, con las necesidades y las posibilidades de toda la sociedad.

Para Olivé:

las ciencias constituyen una parte de la realidad social y consisten en un complejo de actividades, de creencias, de saberes, de valores y normas, de costumbres, de instituciones, etc., todo lo cual permite que se produzcan ciertos resultados ...que se usan para transformar el mundo. (2000, p.3)

En este orden de ideas, se puede inferir, que llamar ciencia a una disciplina, implica evidenciar estos criterios como categorías descriptivas. Y cabe entonces la posibilidad de hablar de ciencias como una forma ortodoxa en que se enmarcan a aquellas disciplinas cuya sistematización precide la manera en que encaran la realidad y sus fenómenos.

Nuñez (2005), citado en Asencio (2014), considera que la ciencia se puede analizar como sistema de conocimientos que modifica la visión del mundo real y enriquece el imaginario social y la cultura; se le comprende como proceso de investigación que permite obtener nuevos conocimientos, y que - a su vez- ofrecen mayor número de posibilidades novedosas para la manipulación de los fenómenos. Además, su impacto práctico y productivo, propicia la propia transformación del mundo y es fuente de riqueza. De esta manera, la ciencia se yergue como una profesión institucionalizada, portadora de cultura y con funciones sociales bien identificadas.

En tanto, la transversalidad, implica puntos de contacto y cruces entre las distintas disciplinas, donde cada una aporta sus problemas, conceptos y métodos de investigación. Está presente entre las disciplinas, en las disciplinas y más allá de las disciplinas. En la revisión sobre el término, las definiciones propuestas mayoritariamene resultan complejas y obligan a repasar históricamente su aparición y uso, y a proponer su irrupción en el mundo de ciencias naturales y sociales.

La transversalidad supone un planteamiento integrador y contextualizado, que promueve cierta conciencia a través de la asimilación de lo interdisciplinar y multidisciplinar, para promover una actitud más creativa. Según Magendzo (2005) la transversalidad engloba muchos conceptos que, principalmente, tienen que ver con las "maneras de entender el mundo y las relaciones sociales en un contexto específico.”(p.28)

David Solano Cornejo (2007) por su parte plantea que:

Los conceptos de transversalidad nos llevan a la definición de temas transversales. Estos son temas que articulan el conocimiento, pero que surgen de la realidad. Los temas transversales son aquellos importantes para el desarrollo, y que se convierten en los ejes sobre los cuales se imparten los conocimientos. (p.52) 
Haciendo eco a su propuesta y considerando el análisis que ocupa este texto, el diseño, sería una disciplina de esta naturaleza. Y el enfoque para entenderla, cobraría un sentido distinto, si se analiza desde esta perspectiva. De tal suerte, que elementos afines, o bien que son el centro de los procesos productivos, sociales y culturales, podrían ser acotados y quizá analizados con otros niveles de profundidad, a través de una visión transversal del diseño, su praxis, sistematicidad y cientificidad (entendida como caracterización formal de la disciplina). Desde esta óptica, el propio desarrollo del diseño, primero como oficio, para luego constituirse como una disciplina profesional enmarcada dentro de los procesos sociales, culturales, económicos y políticos, se instituye a partir de procesos dinamizadores, multi, inter y transdisciplinares. Como lo plantea Nuñez (2005) citado en Asencio(2014):

Una de las características del desarrollo científico de finales del siglo XX y principios del siglo XXI es el incremento de diferentes formas de integración horizontal (trabajo en equipos, multidisciplinariedad, interdisciplinariedad, transdisciplinariedad, entre otras) como recurso necesario para generar nuevos conocimientos y tecnologías; los conocimientos no están en la realidad, los construye el hombre; pero no el hombre aislado y ahistórico, sino el hombre en comunidad, el hombre en sociedad, que supone un diálogo, una relación entre razón y experiencia, entre teoría y empiria (p.553).

El concepto de lo transversal ha tenido una rápida transformación, particularmente en los últimos años, utilizándose como bandera de los cruces y trazos que relacionan disciplinas y alcances. Es símbolo de innovación y se relaciona con una perspectiva cuyo eje de acción trastoca y enmarca procesos mucho más complejos para el análisis y la conceptualización. María Beatriz Quintana (2020) refiere en el Diccionario de Pensamiento Alternativo II, que:

Es un concepto complejo en sus tres dimensiones semióticas. Por su sintaxis se trata de una palabra formada por un lexema (transversal) y un sufijo sustantivo de adjetival (-dad). Lo transversal (adjetivo) del latín Transversarius, simétrico de transversus puesto que atraviesa. Si bien es un concepto básico que se relaciona con la matemática y la geometría ... su aplicación cualitativa nos permite entrar en terrenos epistemológicos y sociológicos de gran alcance en el siglo XX y XXI. Su alcance "cultural” reviste aristas teóricas y prácticas tan diversas, que nos permite pensar al concepto en función de oposiciones, descripciones e incluso fundamentaciones de ciertas actividades que el hombre vive en la época actual (s/p).

En este sentido, y desde una dimensión pragmática, la transversalidad es un concepto que tiene diversos usos contextuales y se aplicaría metafóricamente a cualquier ámbito. Analizar transversalmente, se asocia de manera generalizada, a cruzar relaciones o contextos desde distintas disciplinas, más allá de cruzar una intencionalidad por todas ellas o bien "mirar" desde una perspectiva más polivalente, si esto pudiera calificarse de tal manera. 
Desde el diseño, hablar de transdisciplinariedad en un primer plano, representa implicarlo en una práctica recurrente que lo acerca a una especie mediación, considerándolo el primer eje de acción.

Esta concepción, alude principalmente a que el diseño no se configura como un ente aislado, más bien funge como un punto de convergencia entre una gran diversidad de indicadores, que media para fundamentar sus ejes de acción y los alcances de su intervención. Y en este sentido, resultaría innegable la relación triádica método-tecnología-diseño, enmarcándola a priori a nivel de disciplina ciéntifica, al determinarse con el propio proceso de diseño, sus alcances, dimensiones, impacto, niveles de intervención y dinámicas para la ejecución.

Por lo tanto, resulta oportuno partir de una base conceptual para definir lo que se asume como diseño. En primer término se recupera la definición que Miguel Ángel Herrera (2018) propone en su texto Investigación en diseño: su realidad y objeto de estudio, que sin elevar su definición como alguno único y preciso, tal como el mismo lo indica, la delimita de forma descriptiva como:

la actividad práctica que tiene por objeto desarrollar alternativas de solución a necesidades (básicas, estéticas, de significado, o de otra índole) de un usuario (individual o colectivo) a través de productos (materiales, digitales o espaciales) que son resultado de la actividad proyectual y en los cuales, la experiencia de uso, las características formales y funcionales constituyen rasgos fundamentales, distintivos y definitorios. (Herrera, 2018, p.29)

A partir de su concepción y tal como lo expone, entonces se puede asumir que todo producto de diseño posee una dimensión cultural, su propio lenguaje y además se le asigna una utilidad práctica vinculada con usuarios, individuales o colectivos, a quienes les proporciona una experiencia de uso, un disfrute estético, un signficado y sentido. El término diseño es polisémico y se le asignan infinitud de acepciones e interpretaciones; su etimología latina di-segno refiere a la comunicación a través de signos; $d i$, por medio, a través; segno, signum, objeto, fenómeno o acción material que, por naturaleza o convención, representa o sustituye a otro; indicio, señal de algo, en el idioma italiano disegno es dibujo, asociado fundamentalmente a los principios de visualización-representación.

El diseñador como profesional, interviene en la concepción del mundo para formalizar y dar un servicio útil a empresas y personas, haciendo hincapié en su capacidad para involucrarse en distintos procesos, desde los más técnicos, hasta los más complejos, como los económicos, sociales, culturales, estéticos, psicológicos, etc., definiendo y desarrollando productos y servicios de diseño. Siendo un proceso clave de la comunicación, es importante un conocimiento más profundo de sus alcances y dimensiones dentro de la sociedad. Como actividad inserta en los procesos productivos, tienen repercusiones económicas; desde lo social, impacta de forma positiva y negativa en la sociedad y desde un ámbito cultural, tiene grandes implicaciones de identidad y referencia. Como una disciplina en el contexto contemporáneo puede ser identificada como fenómeno cultural, como práctica social, como acto creativo, como disciplina académica, como actividad productiva, etcétera. 


\section{El diseño como disciplina transversal}

El diseño como disciplina, ligada estrechamente a la academia, y contextualizada en los albores del siglo XX, surge precedida de un amplio y vasto desarrollo tecnológico y científico muy particular y sin precedentes, tal como lo plantea Herrera (2017). Esta recién constituida área se alineó al pensamiento racionalista, relacionado directamente con el positivismo, buscando despegarse del mundo del arte y del concepto de oficio, para iniciar su profesionalización como disciplina. En este sentido, Rodríguez (2012) afirma que:

Cuando alrededor de 1900 se inicia el debate sobre la posibilidad de una ciencia de la proyección de objetos que pudiera ser inscrita dentro del ámbito de las disciplinas de la ingeniería, muchos proyectistas, fortalecidos por la reciente conquista de la industrialización, abandonaron toda relación con el arte, sosteniendo que su labor no consistía en la creación de objetos bellos y significativos, sino puramente funcionales y técnicamente reproducibles. (2012, p.16)

A partir de entoces, el diseño se aleja del arte para acercase a los procesos productivos e industriales y se vincula directamente con la técnica y tecnología como banderas de su propio proceso. Este giro de las disciplinas proyectuales, dentro de las que el diseño se configura, marca el acercamiento primario a la ciencia, pues de ella toma el uso de métodos, técnicas y procedimientos para diseñar, lo que a su vez se institucionaliza también, como parte de la formación de los profesionales en el área.

Escuelas como Ulm (Hochschule für Gestaltung o $H f G$ ) y la emblemática Bauhaus, que surgen en pleno auge de la industria y la producción masiva, determinan la comprensión del diseño como un proceso articulado por una serie de fases ordenadas de forma continua, que inicia con la recolección de datos y culmina con la presentación de un proyecto.

Desde la visión de Tomás Maldonado(1965), la noción de proyecto, implica un proceso racional orientado con un propósito específico vinculado con la sociedad. En su perspectiva, "tanto el hecho como la ficción que rodea a la $H f G$ coinciden en un punto; el énfasis en la metodología del diseño, es decir, el interés en una relación entre la ciencia y el diseño" (p.31). Adicionalmente a estas afirmaciones y apoyado en las aportaciones que sobre el tema propuso Gui-Bonsiepe, desarrollan juntos el texto de 1964 titulado Ciencia $y$ diseño, a partir del cual el método proyectual se rige por procedimientos científicos.

Maldonado fue uno de los primeros en reconocer de forma muy clara que el acelerado desarrollo técnico y económico planteaba nuevas exigencia en la enseñanza del diseño y con ello el modelo educativo de la Bauhaus quedaba obsoleto, pues se había formulado sobre premisas artísticas y no científicas y luego de analizar todas las orientaciones y modelos educativos que aparecieron durante y después de esta escuela, él veía como una solución adecuada para ofrecer una salida eficaz, que se introdujera en la formación del diseñador el "pensamiento científico operacional" cuya principal implicación se orienta hacia establecer una metodología dónde el discurso científico y teórico fueran parte sustantiva. 
Y con esta idea, fue Lászlo Moholy-Nagy quien invitó en los años cuarenta a científicos de la Univesidad de Chicago a que impartieran clases en la llamada Nueva Bauhaus. Disciplinas como cibernética, matemáticas o semiótica, formaron parte del programa de cursos. De esta manera se cubría la necesidad que dictaba el mundo contemporáneo, de un profesional vinculado con las innovaciones científicas y tecnológicas, y también de forma directa, se elevaba la formación del estudiante de diseño.

Esta transformación estructural propuesta por Maldonado, quien reconoció y propició un cambio radical en la forma de enseñar y formar al diseñador, fue motivo de críticas, pero haciendo eco a muchos de los teóricos que lo han estudiado, se insiste en que, ¿qué hubiera pasado si no se hubiera dado este paso tan radical? ¿Dónde se encontrarían hoy muchas escuelas, profesores y estudiantes, si no se hubiera puesto en práctica? Hasta ese momento no existía propuesta alguna que planteara una sistematización del diseño y que a su vez diera paso a lo que hoy, de forma generalizada, se conoce como el proceso formal de diseño (independientemente de las metodologías y escuelas que lo amparan).

A partir de ese momento las disciplinas del diseño, así como se comenzaron a enseñar en HfG Ulm y en la Nueva Bauhaus surgían de actividades de planificación y sistematización, a través de un proceso de diseño, muy distante ya de los calificativos de mero acto creativo, espontáneo o producto de la inspiración. En palabras de Bruno Munari (1977):

La creatividad es indispensable en el campo del diseño, siempre que se entienda el concepto de diseño como proyectación. Este - si bien es libre, como la fantasía- hace uso de los métodos exactos, como la creación (p.26)

Por su parte Herbert Simon(1978) en su libro titulado The Sciences of the Artificial, concibe al diseño como ciencia y plantea la categorización de las ciencias del mundo natural y las del mundo de lo artificial, estas últimas, responsables del estudio y enseñanza de todo aquello creado por el hombre, categoría en la que encaja el diseño.

Su visión del diseño es muy amplia, pues plantea que cualquier acción destinada a cambiar situaciones existentes por situaciones ideales o deseadas, desarrolla diseño. En el texto la Ciencia del Diseño (Simon, 1996), el objetivo fundamental consistió en poner sobre la mesa los argumentos suficientes para definir al diseño como una ciencia. Su propuesta no solo quedó en este nivel, llegó hasta los centros de enseñanza profesional del diseño, haciendo énfasis en el hecho de que las escuelas deben asumir su reponsabilidad en la medida que descubran y enseñen una ciencia del diseño, a través de un cuerpo de pensamiento intelectualmente robusto, analítico, parcialmente formalizable, práctico y empírico al que se sume una doctrina sobre el proceso de diseño.

Analiza la importancia de solucionar los problemas de diseño a partir de herramientas de análisis lógico, criterios de utilidad, eficiencia, optimización, etc., que no se quedan solo en una solución, sino en la búsqueda de alternativas, lo que da soporte a lo que él denomina las Ciencias del diseño. Le resultaba fundamental incorporar distintas herramientas científicas en la enseñanza del diseño, y resaltó la necesidad de plantear objetivos claros y diversos para poder encarar las complejidades propias del diseño en un contexto socio-cultural. 
Adicionalmente también propuso aspectos relevantes, que más adelante se recuperan e institucionalizan en otras escuelas modernas del Diseño. Estos lineamientos, fijan las orientaciones incluso para establecer sus propios métodos o metodologías. Se caracterizan por comenzar con la sistematización, luego con la proyección y planificación del diseño en contexto, y no como un ente aislado. Finalmente otorgan un peso fundamental a la evaluación, para comprobar resultados, medir su eficacia y determinar su incidencia e impacto para los usuarios (la sociedad).

Como lo expresa Herrera (2017), las ideas de Maldonado y Bonsiepe pueden resumirse en el reconocimiento del aporte que la ciencia hace al diseño al proveer mecanismos para eficientar el propio proceso de diseño, sistematizar la información y mejorar el proceso de análisis y síntesis, sin que se afirme categóricamente que se trata de una ciencia. Más bien, se trata de una disciplina cuyas orientaciones toman aspectos relevantes de las ciencias para configurar sus propios procesos. Las relaciones dialógicas entre el diseño, visto como disciplina y visto como ciencia, han favorecido su propia transformación y le han permitido ir madurando. Desde el principio, cuando en el contexto del siglo XX se desplazaba entre las artes y los oficios, hasta caracterizarse disciplinarmente como una profesión, sus desplazamientos superaron el ego natural de una naciente disciplina, para concederse más un compromiso con los afectos, efectos y necesidades de una comunidad, entendiendo su precisa circunstancia dentro de los múltiples procesos de transformación de la vida cotidiana. En este camino de sistematización se enmarca al proceso de diseño, como lo afirma Luz del Carmen Vilchis(2002), en "virtud de una metodología que sirve como puente entre los principios generales del hacer y el conocer, comunes a todo saber con las características específicas de la materia" (p.9). Justamente estos principios metodológicos ponen en evidencia procesos interdisciplinares y transdisciplinares, donde la teoría y la práctica del diseño se conciben, cobran sentido y reafirman la función social inherente a su intervención y vuelven a reforzar su importancia en el centro de los propios procesos de investigación y praxis, como objeto de estudio para el diseñador en formación o el profesional.

Adjetivar al diseño como una disciplina científica puede resultar complejo, quizá no así reconocer su cientificidad al valorar la importancia de sus métodos, técnicas y metodologías. El diálogo, entonces, se centra en el desarrollo de esta racionalidad operativa, dentro de un orden social, cutural, político y económico, que da paso a la modernidad y al desarrollo de la ciencia, desde el renacimiento y hasta nuestros días y que sitúa al diseño como una estructura que entrelazada racionalidad y operacionaldad, técnicas y métodos, y que en su momento devienen en la metodología proyectual, que a su vez dará origen a lo que de forma generalizada se entiende como el proceso de diseño. En este sentido y tal como lo afirma Vilchis (2002), "el diseño se presenta como una disciplina y un hacer integrado en el ámbito social, pues y como todo hacer humano no puede ser desligado de sus condicionamientos, ni de sus consecuencias sociales". (p.10)

De esta manera lo que pudiera ser la ciencia del diseño o más adecuadamente, en virtud de lo revisado en este texto, la cientificidad del diseño, cobría sentido situada en un dialógo extenso y profundo entre lo filosófico, ontológico y epistemológico al que se sumarían la habilitación del profesional para entender y asumir la responsabilidad de su quehacer al momento en que inicia el proceso y la propia orientación de su trabajo al beneficio social, 
no de un individuo-usuario, sino de una comunidad. Asimismo, y como eco a estas interacciones transdisciplinares, atinadamente Aurelio Horta Mesa (2007) precisa que:

Desde Gutemberg, pasando por las primeras exposiciones universales en Viena, Filadelfia o París hasta la Bahaus, y llegando hasta los ordenadores, los procesos del diseño han significado imperativos para la competencia económica, el bienestar social y el ejercicio político. (p. 135)

Una implicación importante dentro de este escenario es que cualquier investigación en el diseño, para el diseño y desde el diseño, está en la obligación de legitimar sus propios modos de abordaje y de resolución de problemas, frente a la gran divergencia de criterios existentes. Además, justo para conseguir esa legitimación, el diseño debería asumir, de forma permanente, una visión a partir de la transversalidad, al estructurar la investigación, definir y declarar las orientaciones del trabajo desde un marco de convicciones epistemológicas y metodológicas, sustentadas en una condición académico-estructural que permita sistematizar su proceso, desde la proyección, hasta la solución. Así, las soluciones se vinculan con las demás disciplinas y las disciplinas se dejan "atrevesar" por el diseño. Es innegable que el diseño nace con una tradición interdisciplinaria, que se va acentuando, de la mano de las tecnologías digitales, hacia la transdisciplinariedad, y de este modo, su relación con otras disciplinas es incuestionable. Más aún, el marco de acción que caracteriza al diseño es inescindible de los cruces entre otros campos, cuando se determina el proceso para la conformación de soluciones.

Los esfuerzos por desarrollar, no solo la práctica, sino la investigación en el diseño, para el diseño y sobre el diseño, han derivado en corrientes y tendencias vinculadas, por ejemplo, a la especialización y a los estudios de posgrado, aunque también han favorecido una práctica profesional distinta, introduciendo a la metodología científica en el desarrollo de los proyectos. Esto ha conseguido que el producto resultante se soporte no solo en principios y fundamentos del diseño, también en datos, métodos y metodologías (para recolección de información, diagnóstico, comparación, etc.) y que las conclusiones obtenidas se resuelvan a través de técnicas de investigación científica procedentes principalmente de las Ciencias Sociales, aunque en algunos casos, también se hacen presentes las ciencias naturales. Entonces, se puede concluir, recuperando lo que Miguel Herrera(2018) afirma:

que siempre será mejor basar la práctica del diseño en principios y teorías apoyadas en la investigación formal. En definitiva, aun cuando el diseño no constituye una ciencia, la investigación sistemática y rigurosa, será siempre de gran utilidad para dar certeza a su práctica y contribuir al desarrollo propio de la disciplina. (p.28)

A poco más de 50 años de que se pusiera sobre la mesa la propuesta del diseño como una ciencia de lo artificial, hoy siguen analizándose y gestándose nuevas reflexiones sobre la forma de hacer e investigar el diseño y, sobre todo, en las maneras de concebirlo y dialogarlo. Interrelacionar, cruzar los bordes e incluso atravesarlos, pone en evidencia que 
del diseño se van desprendiendo ámbitos, alcances, y hasta subdisciplinas que obligan a detenerse y reflexionar sobre la cientificidad de su hacer.

Se delinean así, desde las nuevas formas de concebirlo, organizarlo y abordarlo, hasta nociones fundamentales sobre sus convergencias, divergencias, evoluciones y modificaciones. Además, vemos en la práctica profesional, en el ámbito laboral y en las escuelas, cómo se perfilan entronques y cruces que superan las tradiciones conceptuales, para situar al diseño en relación directa con la Ciencia.

Hay quienes han hablado de la ciencia del diseño, otros más que distinguen entre diseño y ciencia y en ese camino, los cruzan, acercan o alejan, lo cierto es que ambos son analizados de manera dialógica, recíproca, no limitativa. Estos abordajes conducentes a determinar elementos de distinción, unión, intencionalidad y postura, vuelven la reflexión al mundo de la epistemología y permiten afirmar que de forma axiológica, metodológica, estratégica y operativa, la praxis del diseño moviliza a un plano categórico que sin ser del todo ciencia, lo acerca a una Ciencia del diseño o al Diseño con una carectirización científica.

\section{Reflexiones finales}

Como señala De Bont: "Dada la creciente complejidad de las cuestiones sociales, los diseñadores desempeñan un papel vital y central en la innovación basada en el diseño. Esto requiere conocimiento: metodología, herramientas y nuevos conceptos relativos a los usuarios, tecnología y aspectos empresariales" (2013: 2), lo que de alguna manera acerca al diseño a la ciencia.

Es por ello que la investigación sistemática en diseño se vuelve una necesidad imperante no sólo en los espacios académicos, o como parte de la formación en escuelas y universidades, también en el ejercicio libre de la profesión y en la propia profesionalización de la disciplina.

Elevarlo al carácter de ciencia cobra significado si primero se profundizan, desde órdenes filosóficos y epistemológicos profundos, y se vuelve a pensar al diseño desde su configuración, desde el contexto que lo enmarca históricamente y de las implicaciones de su intervención. De allí que sería deseable que se validen distintos órdenes disciplinares. Quizá entonces, en vez de proliferar subdisciplinas del diseño, especialidades o hibridaciones ambiguas, se comience a caracterizar la ciencia del diseño atravesando y cruzando las fronteras que hoy lo limitan.

Bajo estos supuestos y haciendo eco de lo que Joan Costa declaró atinadamente en su reflexión El futuro del diseño: “...en este momento en que la crisis del diseño procede de que hay en él demasiada tecnología, poca metodología y nada de filosofía, deberíamos reflexionar sobre el origen y los principios del diseño. Una mirada a los orígenes..." (Costa, 2008, p.2)

Y se podría cerrar esta reflexión, afimando que el diseño se caracteriza por su cientificidad y que el proceso de diseño, está estrechamente vinculado con métodos y metodologías propias del hacer profesional, la investigación y generación de conocimiento novedoso, 
que lo encuandran y perfilan hacia la sistematicidad, sin que con ello se hable formalmente de una ciencia, o no al menos, de lo que tradicionalmente se reconoce como eso. El diseño ha dejado de ser tan solo oficio y profesión, para institucionalizarse como una disciplina que transversaliza la comunicación y las formas en que se gesta esa comunicación. El diseño opera en una cultura que se caracteriza por la digitalización y las tecnologías informáticas, que se nutre de la innovación y todo lo que ello implica y que se complementa con una práctica situada en el contexto de necesidades claras de la sociedad. Por ello, como lo referie Costa (2008):

El Diseño necesita humanizarse. El Diseño puede y debe hacerlo. Él posee la capacidad dinámica de socialización por medio de los objetos y los mensajes que se relacionan con los individuos. Y la aptitud por construir y difundir conocimiento, es decir, cultura. Si nuestra época se define con estas cuatro ideas: era de la Comunicación, economía de la Información, cultura de Servicio y sociedad del Conocimiento, entonces, éstos son los cuatro puntos cardinales que han de orientarnos hacia el futuro del Diseño. Y el diseño del Futuro. (p.11)

Sólo mediante una amplia discusión sobre aspectos epistemológicos, ontológicos y metodológicos, se podrá perfilar una verdadera ciencia del diseño. El diálogo no ha concluido, incluso parece aún más amplio y polisémico, puesto que sobre su base descansan, al estilo de John Berger (1972), muchos modos de ver.

\section{Bibliografía}

Ander-Egg, E. (1995). Técnicas de investigación social (Vol. 24). Buenos Aires: Lumen.

Asencio, E. (2014). Una aproximación a la concepción de ciencia en la contemporaneidad desde la perspectiva de la educación científica, Ciência \& Educação (Bauru), vol. 20, núm. 3, 2014, pp. 549-560 Universidade Estadual Paulista Júlio de Mesquita Filho São Paulo, Brasil

Costa, J. (2008). El futuro del diseño, Disponible en : https://www.academia.edu/5690780/ Futuro_diseno

Gamoral, R. La disciplina del Diseño desde la perspectiva de las Ciencias Sociales. Disponible en :https://www.researchgate.net/publication/250310963_La_disciplina_del_Diseno_desde_la_perspectiva_de_las_Ciencias_Sociales [Consultado Mar 25 2021].

Kröber, G. (1986) Acerca de las relaciones entre la historia y la teoría del desarrollo de las ciencias. Revista Cubana de Ciencias Sociales, La Habana, v. 4, n. 10, p. 27-32, 1986.

Herrera, M. (2017). Diseño: entre el diseño científico y las ciencias de lo artificial. En: No Solo Usabilidad, no 16, 2017. Disponible en: http://www.nosolousabilidad.com/ articulos/diseno_cientifico.htm\#: :text=Al\%20respecto\%20Rodr\%C3\%ADguez\%20 (2012)\%2C,reciente\%20conquista\%20de\%20la\%20industrializaci\%C3\%B3n\%2C 
Herrera, M. (2018). Investigación en diseño: su realidad y objeto de estudio. Disponible https://www.researchgate.net/publication/349534498_Investigacion_en_diseno_su_ realidad_y_objeto_de_estudio

Horta, A. (2007). Actas de Diseño No. 2, Universidad de Palermo, Disponible en: https:// doi.org/https://doi.org/10.18682/add.vi2

Magendzo, Abraham (2005), "Currículo y transversalidad: una reflexión desde la práctica", Revista Internacional Magisterio, educación y pedagogía, № 16, Bogotá.

Méndez, C. E. (1998). Metodología. Guía para Elaborar Diseños de Investigación en Ciencias Económicas, Contables y Administrativas, Bogotá: McGraw-Hill.

Maldonado, T. (1958). Nuevos desarrollos en la industria y en la formación del diseñador de productos, ULM, 2, octubre de 1958.

Munari, B.(1983). Cómo nacen los objetos, Apuntes para una metodología proyectual, España:Gustavo Gili.

Nuñez, J.(2005).La ciencia y la tecnología como procesos sociales, La Habana:MINED.

Olivé, L.(2000). El bien, el mal y la razón. Facetas de la ciencia y de la tecnología. Capítulo 1. ¿Qué es la ciencia? UNAM: Paidós.

Padrón, J. (2000). La Estructura de los Procesos de Investigación, Revista Educación y Ciencias Humanas, 15, 7-12.

Padrón, J., \& Camacho, H. (2000). ¿Qué es Investigar? Una Respuesta desde el Enfoque Epistemológico del Racionalismo Crítico. Telos, 2 (2), 314-330. Padrón, J. (2006). Notas sobre cantinflerismo académico. Disponible en: https://www.researchgate.net/profile/ Jose_Padron-Guillen/ Fecha de consulta: 2 de marzo de 2018. Padrón, J. (2007). Tendencias epistemológicas de la investigación científica en el siglo

Padrón, J. (2014). Notas sobre enfoques epistemológicos, estilos de pensamiento y paradigmas: Proyecto de Epistemología en DVD. Doctorado en Ciencias Humanas, Maracaibo: Universidad del Zulia.

Quintana, M. B. (2020). Transversalidad, Diccionario del Pensamiento Alternativo II, Disponible en: http://www.cecies.org/articulo.asp?id=250.

Rodríguez, L. (2012) El diseño y sus debates: Antologías. Ciudad de México: Universidad Autónoma Metropolitana, Unidad Xochimilco.

Simon, Herbert (1996). The Sciences of the Artificial. (Third edition) Cambridge: The MIT Press. Massachusetts Institute of Technology.

Solano, D. (2007). La transversalidad y transectorialidad en el sector público, ¿Cómo enfrentar los desafíos de la transversalidad y de la intersectorialidad en la gestión pública?, XX Concurso del CLAD, Disponible en: https://www.researchgate.net/search.Search. html?type $=$ researcher\&query $=$ david $\% 20$ solano

Vilchis, L.(2002). Metodología del Diseño. Fundamentos Teóricos, México:Claves Latinoamericanas. 


\begin{abstract}
The epistemology of design has focused its efforts on treating the discipline as an object of study and framing it within the framework of sciences. The main intention has been to understand it and make it understood as a scientific activity, approach it as a discipline and place it in the academic context. In this way, the design is instituted as an object of study, but also as a subject: historical, social, cultural, political, semiotic and linguistic, on which basic assumptions that would allow its scientific validity to be recognized.

As a discipline, whose origin is located in the twentieth century, its approach to science was widely manifested in the use of design methods, procedures, technologies and scientific techniques, as well as in the professional practice and training of designers. It has evolved and allowed us to see that it also uses other sciences, to show inter or multidisciplinary processes that converge in its approach. Analyzing the approach to its praxis, as a discipline of its time, it has been framed in a perhaps intentional or even daily transversality. In this way, it crosses other disciplines to determine assumptions that transcend beyond the professional and economic sphere and allow it to become a phenomenon of analysis that affects all aspects of our society and daily life.

The relationship between design and science was raised by Herbert Simon in the seventies of the twentieth century, considering design as a science. His position exposes the need to scientifically study the world created by man and look at design as a science of the artificial. This article offers a proposal, which analyzes the design process, its methods, techniques, technologies and products, in order to put on its scientificity and its scope as a discipline that cuts across the rest.
\end{abstract}

Keywords: design - diálogo - disciplina - ciência - transversal

Resumo: A epistemologia do design concentrou seus esforços em tratar a disciplina como objeto de estudo e enquadrá-la no quadro das ciências. A intenção principal tem sido compreendê-la e fazê-la compreender como atividade científica, abordá-la como disciplina e inseri-la no contexto acadêmico. Desse modo, o design se institui como objeto de estudo, mas também como sujeito: histórico, social, cultural, político, semiótico e linguístico, sobre os quais pressupostos básicos que permitiriam reconhecer sua validade científica.

En quanto disciplina, cuja origem se situa no século XX, a sua abordagem à ciência manifestou-se amplamente na utilização de métodos, procedimentos, tecnologias e técnicas científicas de design, bem como na prática profissional e formação de designers. Evoluiu e permitiu-nos ver que também utiliza outras ciências, para mostrar processos inter ou multidisciplinares que convergem na sua abordagem. Analisando a abordagem de sua práxis, como disciplina de seu tempo, ela se enquadrou em uma transversalidade talvez intencional ou mesmo cotidiana. Desse modo, atravessa outras disciplinas para determinar premissas que transcendem a esfera profissional e econômica e permitem que se torne um fenômeno de análise que afeta todos os aspectos de nossa sociedade e do cotidiano. 
A relação entre design e ciência foi levantada por Herbert Simon nos anos setenta do século XX, considerando o design como uma ciência. Sua posição expõe a necessidade de estudar cientificamente o mundo criado pelo homem e olhar o design como uma ciência do artificial. Este artigo oferece uma proposta que analisa o processo de design, seus métodos, técnicas, tecnologias e produtos, de forma a mostrar sua cientificidade e seu alcance como disciplina que perpassa as demais.

Palavras chave: design - diálogo - disciplina - ciência - transversal

[Las traducciones de los abstracts fueron supervisadas por el autor de cada artículo] 